\title{
Sintomas internalizantes, externalizantes e relações interpessoais de adolescentes em tratamento para dependência química
}

\section{Symptons internalizing, externalizing and interpersonal relations teenagers in treatment for addictions}

\section{Síntomas internalizantes, externalizantes y relaciones interpersonales de adolescentes en tratamiento de adicciones}

\section{Dhiordan Cardoso da Silva*}

Pontifícia Universidade Católica do Rio Grande do Sul - PUCRS, Porto Alegre, Rio Grande do Sul, Brasil

\section{Raquel de Melo Boff**}

Pontifícia Universidade Católica do Rio Grande do Sul - PUCRS, Porto Alegre, Rio Grande do Sul, Brasil

\section{Maria Isabel Wendling***}

Pontifícia Universidade Católica do Rio Grande do Sul - PUCRS, Porto Alegre, Rio Grande do Sul, Brasil

\section{Margareth da Silva Oliveira****}

Pontifícia Universidade Católica do Rio Grande do Sul - PUCRS, Porto Alegre, Rio Grande do Sul, Brasil

\begin{abstract}
RESUMO
O uso de drogas na adolescência produz importantes impactos psicológicos, comportamentais e nos estilos interpessoais do individuo. Este estudo visou examinar e descrever tais aspectos através do instrumento Young Self Report (YSR), uma entrevista clínica semi-estruturada e uma sessão de grupo focal com cinco adolescentes internados pela a dependência de drogas. O discurso dos participantes foi analisado através da Análise de Conteúdo de Bardin, modalidade temática e as sintomatologias clínicas examinadas pelo YSR. O conteúdo do grupo obteve seis categorias finais: habilidades interpessoais, dificuldades interpessoais, aspectos emocionais, aspectos físicos, ambiente escolar e ambiente familiar. Os resultados mostraram características importantes no funcionamento adaptativo, que facilita as relações e problemas clínicos que podem estar associados a estilos menos adaptativos. Este estudo destaca a importância de avaliar os elementos individuais envolvidos na dependência das drogas e no plano terapêutico, bem como, sugerir modelos de intervenções que atendem a essas demandas.
\end{abstract}

Palavras-chave: adolescente, transtornos relacionados ao uso de substâncias, aspectos psicológicos, relações interpessoais. 


\begin{abstract}
Drug use in adolescence produces significant psychological impact, behavioral and interpersonal styles of the individual. This study aimed to examine and describe these aspects through the instrument Young Self Report (YSR), a semi-structured clinical interview and a focus group session with five teenagers hospitalized for drug addiction. The speech of the participants was analyzed by the Bardin Content Analysis, thematic modality and clinical symptomatology examined by $Y S R$. The content of the group obtained final six categories: interpersonal skills, interpersonal difficulties, emotional, physical, school environment and family atmosphere. The results showed important characteristics in the adaptive operation which facilitates relations and medical problems which may be associated with less adaptive styles. This study highlights the importance of assessing the individual elements involved in drug addiction and therapeutic, as well as suggest intervention models that meet these demands.
\end{abstract}

Keywords: adolescent, substance-related disorders, psychological aspects, interpersonal relations.

\title{
RESUMEN
}

El consumo de drogas en la adolescencia produce impacto psicológico significativo, estilos de comportamiento e interpersonales del individuo. El objetivo este estudio fue examinar y describir los aspectos através del instrumento Young Self Report (YSR), una entrevista clínica semiestructurada y una sesión de grupo focal con cinco adolescentes hospitalizados por adicción a las drogas. El discurso de los participantes fue analizado por el análisis de contenido de Bardin, modalidad temática y la sintomatología clínica examinadas por YSR. El contenido del grupo contenida seis categorías: habilidades interpersonales, dificultades interpersonales, ambiente emocional, físico, la escuela y ambiente familiar. Los resultados mostraron características importantes en el funcionamiento adaptativo que facilita las relaciones y problemas clínicos que pueden estar asociados con los estilos de menos de adaptación. Este estudio menciones la importancia de evaluar los elementos individuales implicados en la adicción de drogas y terapéutica, así como proponer modelos de intervención que cumplen estas exigencias.

Palabras-clave: adolescente, trastornos relacionados con sustancias, aspectos psicológicos, relaciones interpersonales.

\section{Introdução}

A adolescência é um período marcado por importantes transições. Entre elas, destacam-se as mudanças no corpo, nas relações interpessoais e nos aspectos emocionais (Blakemore \& Mills, 2014; Schoen-Ferreira, Aznar-Farias \& Silvares, 2003), assim como, significativas transformações no desenvolvimento do cérebro e da maturação (Bava \& Tapert, 2010).

Para a Organização Mundial da Saúde, a adolescência conceitua-se como um intervalo biológico, psicológico e social compreendido entre os 10 e os 19 anos (World Health Organization [WHO], 2011). Cabe destacar, que nessa fase da vida, as discussões acerca da identidade - o conjunto de crenças e padrões comportamentais reunidos - e as 
manifestações no âmbito social e individual são mais frequentes (Habigzang, Diniz \& Koller, 2014). As múltiplas curiosidades e expectativas, atitudes mais impulsivas e eventos de diferenciação, por exemplo, é comumente observado nesta etapa do desenvolvimento. Estudos apontam que é na adolescência, inclusive, que ocorre o primeiro contato com as drogas (Peeters, Vollebergh, Reinout \& Field, 2014).

Conforme os dados da Fundação Oswaldo Cruz (FIOCRUZ, 2013) entre os 370 mil usuários de crack e/ou similares estimados, cerca de $14 \%$ são menores de idade. As dificuldades financeiras graves, a falta de uma alimentação adequada, morte de algum membro familiar, assim como, a violência intradomiciliar, caracterizam fatores de risco e possíveis preditores ambientais e comportamentais para a dependência química na vida adulta (Gilbert et al. 2009; Laranjeira, Madruga, Viana, Pinsky \& Mitsuhiro, 2014).

Sobre as repercussões do consumo recorrente de droga, a perda de sono, atrasos nas funções cognitivas, alterações neurobiológicas e na regulação afetiva são alguns dos problemas relacionados, podendo acarretar importantes perdas na qualidade de vida dessa população (Hasler, Soehner \& Clark, 2014; Peeters et al., 2014).

No campo das relações, as amizades exercem diversas influências de escolhas dos jovens (Schoen-Ferreira et.al., et al., 2003). As expectativas, as atitudes e o desempenho nas habilidades sociais, ajudam a proteger o adolescente no desenvolvimento de problemas internos e relacionais no futuro (Caballo, 2003; Wagner \& Oliveira, 2009).

As dificuldades no funcionamento interpessoal, os problemas externalizantes (Achenbach, \& Rescorla, 2003), ou seja, aspectos não adaptativos nas relações familiares, amizades e profissionais, foram citados em estudos com jovens dependentes de drogas e internados para tratamento (Sayago, Lucena-Santos, Horta \& Oliveira, 2014; Cerutti, Ramos \& Argimon, 2015). Os problemas internalizantes (Achenbach, \& Rescorla, 2003) ou sintomas emocionais e comportamentais, que na evolução do quadro pressupõem condições psicopatológicas, estão frequentemente associados à dependência de substâncias (McClernon \& Kollins, 2008; Degenhardt et al., 2013; Copeland, Rooke \& Swift, 2013; Mangerud, Bjerkeset, Holmen, Lydersen \& Indredavik, 2014).

A partir do que foi exposto e sobre a permanente relevância do tema, esta pesquisa teve o objetivo de analisar o conteúdo do grupo focal com adolescentes internados por dependência de drogas sob as temáticas da identidade, relacionamentos interpessoais e da dependência química e verificar os sintomas internalizantes e externalizantes desses indivíduos. 


\section{Método}

Trata-se de uma pesquisa qualitativa de cunho exploratório. O corpus de análise foi composto pelos dados de uma entrevista semiestruturada, da aplicação do instrumento de medidas sintomatológicas Young Self Report - YSR (Achenbach, \& Rescorla, 2003) e pela perspectiva da análise de conteúdo, modalidade temática (Bardin, 2009), do grupo focal. As discussões temáticas foram desenvolvidas sobre as concepções da identidade, relacionamentos interpessoais e dependência de drogas.

\subsection{Participantes}

Participaram desse estudo cinco adolescentes, com idade entre 15 e 17anos $(M=15,60 ; D P=0,836)$, todos do sexo masculino, que estavam abstinentes do uso de drogas e internados, por medida involuntária, em uma instituição de tratamento para dependência química da região de Porto Alegre, RS/Brasil.

\subsection{Instrumento}

Os participantes responderam uma entrevista semi-estruturada a fim de caracterizar a amostra sobre o histórico do uso de drogas e dos tratamentos realizados anteriormente. Além do roteiro, responderam ao Young Self Report -YSR (Achenbach, \& Rescorla, 2003), que é um inventário de comportamentos, auto-aplicável, para jovens dos 11 aos 18 anos, derivado do Child Behavior Check List - CBCL (Achenbach, \& Rescorla, 2003). Foi estruturado para obter respostas do adolescente a respeito de suas próprias competências e problemas. Permite ao avaliador traçar um perfil comportamental do adolescente, derivado da análise de nove agrupamentos de itens ou síndromes: isolamento, queixas somáticas, ansiedade e depressão (comportamentos internalizantes), problemas de atenção, pensamento, comportamentos agressivos, delinqüentes e intrusivos (comportamentos externalizantes) (Achenbach, \& Rescorla, 2003).

\subsection{Procedimentos para coleta de dados}

A coleta de dados foi realizada em uma clínica de tratamento para dependência química masculina de Porto Alegre - RS/Brasil, por método de conveniência, em dois momentos. No primeiro encontro, os participantes, individualmente, foram convidados a responder a entrevista e ao YSR. Após sete dias, realizou-se um grupo focal, com duração de uma hora e meia, que consistia em três categorias temáticas: a) aspectos da sua identidade, b) relacionamentos interpessoais e c) dependência de drogas. 


\subsection{Análise dos dados}

Os dados sociodemográficos e a escala $Y R S$ foram processados no Statistical Package for Social Sciences (SPSS), versão 19.0, cuja análise foi realizada por meio de estatística descritiva (frequências, médias e desvio padrão). A sessão de grupo foi gravada e transcrita na íntegra, a priori, para o tratamento e a identificação das categorias temáticas (Bardin, 2009). A posteriori, as verbalizações foram desdobradas em subcategorias.

\subsection{Aspectos éticos}

O projeto foi aprovado pelo Comitê de Ética da universidade em que foi conduzido (CEP 735.418). Os participantes foram informados do estudo e posteriormente assinaram o Termo de Consentimento Livre e Esclarecido (TCLE). A fim de preservar o anonimato na pesquisa os participantes foram identificados com a letra $\mathrm{P}$ (sugerindo o termo paciente) e com a sequência do número 1 ao 5 .

\section{Resultados}

Dos participantes, dois deles haviam buscado tratamento pela dependência de cocaína, dois pela maconha e um pelo uso problemático de crack. A tabela 1 apresenta os dados sociodemográficos e sobre o consumo de drogas. 
Tabela 1. Dados sobre as características sociodemográficas e sobre o consumo das drogas.

\begin{tabular}{lllr}
\hline Dados sociodemográficos e de consumo & N & M & DP \\
\hline Idade & 5 & 15,60 & 0,83 \\
Ensino fundamental incompleto & 5 & 1,00 &, 00 \\
Renda familiar (em salários mínimos) & 5 & 3,40 & 1,51 \\
Estado civil solteiro & 4 & 1,80 & 1,78 \\
Atividade remunerada (antes da internação) & 5 & 1,00 &, 00 \\
$\quad$ Sim & 5 & 1,00 &, 00 \\
Estudava (antes da internação) & & 1,60 &, 54 \\
Sim & 2 & & \\
Não & 3 & & \\
No de intemações (no passado) & 5 & 1,20 & 0,83 \\
Tempo no atual tratamento (em dias) & 5 & 66 & 25,10 \\
Tempo de abstinência (em dias) & 5 & 78 & 34,20 \\
Primeiro uso (em anos) de: & & & \\
Tabaco & 5 & 11 & 2,00 \\
Alcool & 5 & 12 & 1,64 \\
Alucinógenos & 2 & 14 & 1,15 \\
Maconha & 3 & 12 & 1,14 \\
Cocaína & 4 & 13 & 2,16 \\
Crack & 3 & 14 & 1,15 \\
\hline
\end{tabular}

Nota. Dados da analise estatística descritiva: $M=$ média; $D P=D$ esvio Padrão.

Os principais motivos que os levaram a experimentar as drogas foram as "curiosidades" e os "sentimentos negativos" do tipo solidão, tristeza e ansiedade. Prejuízos nas relações familiares, nos estudos, nas atividades ocupacionais e com a própria saúde foram os principais danos causado pelo uso. Antes da internação, dois adolescentes moravam com as suas mães, um com o seu pai, um com a esposa e a avó e o outro em um abrigo. Pelo menos um membro da família dos entrevistados fez, ou fazia, uso de drogas.

Os resultados do YSR apontaram importantes aspectos clínicos na amostra estudada. Os dados relacionados ao funcionamento adaptativo ou aos problemas de comportamentos do grupo investigado estão apresentados na tabela 2 . 
Tabela 2. Resultados das escalas sindrômicas do $Y S R$ em cada área avaliada.

\begin{tabular}{|c|c|c|c|}
\hline \multirow{2}{*}{ Área avaliada $(n=5)$} & $\begin{array}{l}\text { Faixa } \\
\text { clínica }\end{array}$ & $\begin{array}{c}\text { Faixa } \\
\text { normal }\end{array}$ & \\
\hline & M & $\mathbf{M}$ & DP \\
\hline Escala sindrômica $(n=5)$ & & & 6,426 \\
\hline Ansiedade e depressão & - & 57,60 & 6,426 \\
\hline Isolamento e depressão & - & 60,00 & 5,366 \\
\hline Problemas somáticos & - & 60,80 & 9,257 \\
\hline Problemas sociais & - & 63,00 & 9,027 \\
\hline Problemas de pensamento & - & 63,20 & 5,215 \\
\hline Problemas de atenção & - & 59,60 & 12,218 \\
\hline Quebras de regras & 81,00 & - & 7,516 \\
\hline Comportamento agressivo & 69,00 & - & 14,474 \\
\hline \multicolumn{4}{|c|}{ Escalas orientadas pelo DSM-IV $(n=5)$} \\
\hline Ansiedade & - & 58,00 & 6,595 \\
\hline Depressão & - & 64,00 & 6,798 \\
\hline TDAH & - & 58,00 & 10,074 \\
\hline Problemas somáticos & - & 60,20 & 6,496 \\
\hline Desafiador de oposição & 66,20 & - & 10,802 \\
\hline Problemas de conduta & 75,20 & - & 13,103 \\
\hline Total de problemas internalizantes & 60,60 & - & 6,767 \\
\hline Total de problemas extemalizantes & 74,40 & - & 10,163 \\
\hline Total de problemas & 68,00 & - & 8,306 \\
\hline
\end{tabular}

Nota. Dados das análises estatísticas descritivas: $\mathrm{M}=$ média; $\mathrm{DP}=$ desvio padrão.

A análise de conteúdo do discurso obtido através do grupo temático foi organizada em significados comuns, gerando seis subcategorias: habilidades interpessoais, dificuldades interpessoais, aspectos emocionais, aspectos físicos, ambiente escolar e ambiente familiar que estão descritas no quadro abaixo: 
Quadro 1. Quadro matricial das categorias obtidas através da análise de conteúdo. CATEGORIA Definição

\section{HABILIDADES INTERPESSOAIS}

Nessa categoria estão descritas qualidades individuais do funcionamento interpessoal, características que favorecem as relaçōes interpessoais. Observamos um repertório socialmente habilidoso, através da identificação de comportamentos e atitudes saudáveis no quadro dos relacionamentos.

Exemplo de "Eu sou um cara legal, assim, sou amigo de todo mundo, eu gosto de Verbalizaçôes conversar, eu gosto de brincar" $(P 1)$. "Sou responsável agora, tranquilo, faço as coisas certas, tenho familia, tenho hora pra levantar, pra dormir" (P2). "Sou companheiro, quando o cara tá chorando eu chego junto do cara pra ver qual é que era né, pra ver se o cara precisa de algo né, do apoio né, não dá pra deixar o cara sozinho né meu, porque quando eu fico chorando ninguém me deixa sozinho né meu" (P3). "Sei lá, acho que sou bonzinho, aceito muito as coisas, não dou bola, tenho uma sobriedade" (P4).

\section{CATEGORIA Definição}

Exemplo de verbalizações

\section{CATEGORIA Definição}

Exemplo de verbalizações

\section{CATEGORIA Definição}

\section{Exemplo de} verbalizaçöes

\section{DIFICULDADES INTERPESSOAIS}

A maioria relatou ter problemas nos relacionamentos. Neste grupo predominaram características que comumente podem prejudicar as relações, tornando o funcionamento interpessoal menos adaptativo. Também é possivel verificar dificuldades no controle dos impulsos.

"Seguinte, grito pra caramba, os caras falam que grito pra caramba mas é o meu jeito, não vou conseguir mudar, é o meu jeito, eu gritava na baia, vou gritar aqui também, minha mãe quis me botar aqui, eu vou gritar aqui também, porque é meu jeito de ser, eu gosto de gritar, é o meu jeito, e vou continuar gritando" (P3). "Me dá sangue nos olhos, se eu não tó no dia certo, já me dá uma raiva, não tem arreganho" (P5).

\section{ASPECTOS EMOCIONAIS}

Predominaram as questões emocionais (ansiedade, estresse, raiva e alegria), que podem estar relacionadas às mudanças internas, muitas vezes impactadas pelos ambientes que frequentam, o desempenho nas interações sociais entre outros.

"Ah, eu sou um cara legal, meio estressado assim né" (P1). "Estava ansioso, me irritei um pouco também, peguei um pedaço de pau, já queria dar um pedaço de pau no P5 também" (P3). "Bah quando tó aqui dentro até esqueço do passado" (P5). "Pô, juro que não chorei, nunca chorei até hoje a morte do meu coroa, eu chorei só uma vez com a minha tia lá que desabafei com ela" (PS). "Quando olho a pessoa já penso em fazer um monte de coisa com ela, tipo um psicopata" (P5). "Quando era pia tinha vários traumas, minha vida foi um trauma, minha vida até hoje é um trauma" (P5).

\section{ASPECTOS FÍsICOS}

$\mathrm{Na}$ entrevista solicitamos que descrevessem como se percebiam. É possivel observar uma ênfase nas características relacionadas ao corpo, aos aspectos físicos e não às questões mais subjetivas, de reflexão ou de personalidade e temperamento, por exemplo.

"Eu me vejo gordinho, eu vejo que tenho que emagrecer, eu me vejo moreno pá, me vejo essas marcas aqui (paciente se refere a cicatrizes no rosto), essas aqui ó das rateadas que eu dei" (P3). "Bah, eu quando me olho tento ver bah como eu era feio, tó cada vez mais bonito agora, bah" (P5). "Ah quando eu saio sim, ah eu gosto de sair bem arrumado, eu nâo gosto de sair mulambão; eu não gosto muito do jeito que eu sou" (P1). "Bah quando eu me olho no espelho, bah, tá loco, tu não viu quando eu cheguei, bah, pó tá loco engordei bastante, tô melhor, tô com barriguinha, dai eu olho, bah, tô com esse bigodinho... ah eu queria ficar com bigode, com mais cara de homem, tenho uma cara de gurizinho" (P5). 


\section{CATEGORIA Definição}

Exemplo de verbalizações

\section{CATEGORIA Definição}

\section{Exemplo de verbalizações}

\section{AMBIENTE ESCOLAR}

Todos mencionaram que o espaço escolar seria um ambiente de grande circulação e consumo de drogas, bem como, um espaço de manifestações violentas, relações de amizades e namoros.

"Bah, eu me dava bem com todo mundo... várias minas, vários baseados... eu estudava de noite, às seis horas, chegando à frente do colégio, os moleques tudo fumando maconha, eu dava umas bandas com os caras, dai depois eu ficava lá, com os olhos vermelhinhos né, comia vários Mentos né, pra disfarçar por que a diretora queria sentir o cheiro né, eu passava perfume, fumava um cigarro né, depois de um "beck" né, depois entrava pra dentro" (P3). "Eu achava a escola normal, eu não falava com os pequenos, sempre me dei com pessoas mais velhas, brincava com pessoas mais velhas, tinha medo de machucar os mais novos... no colégio eram tudo pia" (P4). "Bah, na escola eu só ia chapado de maconha, fumava um baseado e ia pra escola, era assim, chapado" (P2). "Eu até matava aula" (P5). "Ah, minha relação era afu, me dava com todo mundo, mas a parte que eu mais gostava era das brigas ... eu estudava, fazia tudo direitinho, a parte da droga eu nem gosto de falar muito, mas as brigas assim era afu, era legal, voava cadeira e classe pra tudo que é lado... eu tinha várias minas também" (P1).

\section{AMBIENTE FAMILIAR}

Muitas queixas e sentimentos de tristeza, solidão e vazio construíram essa categoria. Os estilos parentais, a dificuldade de construir e manter vínculos, assim como, situaçôes de maus tratos e abandono foram questões de reflexão sobre as suas familias. O afastamento da família também surgiu no relato dos entrevistados.

"A minha vida sempre foi um córrego, morando aqui, morando com o outro, nunca tive. Nunca pude mandar em mim mesmo, os outros me atiravam em qualquer canto como se eu fosse um lixo, isso que me dá uma raiva, daí por isso eu não namorava, dá eu vou ficar uma mina, quando về amanhã tá me mandando embora. A minha mãe, olha o que ela fez pra mim, me deixou aqui como um lixo, hoje falo com ela normal, nunca julguei ela, ela veio aqui, e falei na cara dela e falei: ó mãe, tu é isso, tu é isso e tu é isso, eu sempre segurei aqui ó, segurei no peito aqui, a minha mãe se morrer não vai fazer falta. eu não consigo achar um momento feliz que vivi com a minha mãe, não consigo catar um" (PS). "É difícil né cara, bah, olha o que meu pai fez comigo né meu, bah, meu pai parece que sou um lixo pra ele, me tocou no canto e seguinte, vai com a tua mãe e te some; é claro que foi um abandono. Meu, eu apanhava de tudo da minha mãe, de paulada, de facão, daqueles pauzão assim $\delta$, na cabeça, e eu nunca encostei um dedo nela" (P3). "Meu pai tentou me dar um facão, já tentou me dar uma paulada, já me bateu, me deixou todo roxo. Lá em casa não tem água nem luz pra nois, nunca tive uma familia normal, até hoje eu me pergunto por que eu não tive uma mãe, porque eu não tive uma familia normal. Eu não gosto de não ter tido uma família, eu não tive um pai, não tive uma mãe, nem irmão" (P4). "Sinto saudades dos meus primos e do meu irmão. Eles foram meio arrogantes, mas eles săo tri, sinto saudades" (P5). "Eu sinto falta do meu pai também" (P4). "O importante pra mim é minha família. Eu gosto mais da minha avó e da minha mulher" (P2). "O que é mais importante pra mim é minha família né, meu pai, minha mãe, o que eu tinha de mais importante e hoje eu não tenho mais né; nem quero comentar muito sobre" ( $P 1)$.

\footnotetext{
"Organização do material obtido no grupo com as temáticas: a) identidade, b) relacionamentos interpessoais e c) dependência química.
} 


\section{Discussão}

Os aspectos relacionados ao consumo das drogas, como a média de inicio do uso de drogas, o abandono dos estudos e a prática de atos infratores, esteve presente no relato dos participantes, aspecto este que confirma uma tendência observada em outros estudos com a mesma população (Sanchez \& Nappo, 2002; Martins \& Pillon, 2008; Lucena \& Ferreira, 2011).

Quanto ao estado emocional antes do uso de drogas, a maioria relatou estados de sentimentos negativos, como tristeza, ansiedade e solidão sendo um fator importante e que influenciou a primeira experiência do uso de drogas. Entre os prejuízos relatados, foi unânime a identificação de problemas criados nas relações familiares, nos estudos, atividades profissionais e com a própria saúde. Estes resultados também foram encontrados em outros estudos que analisaram fatores associados ao funcionamento emocional, situações e comportamentos gatilhos e histórico de uso de drogas de adolescentes (Pechansky, Szbot \& Scivoletto, 2004; Mombelli, Marcon \& Costa, 2010; Ferreira, Capistrano, Maftum, Kalinke \& Kirchhof, 2012).

Conforme a avaliação de sintomatologias, através do YSR, os achados clínicos destacaram problemas externalizantes, como comportamentos agressivos e de quebra de regra. Tais sintomas e comportamentos podem ser comuns, de forma transitória, na infância ou adolescência, ou ainda, evoluir para um quadro de transtornos de personalidade na idade adulta (Hart, Cox \& Hare, 1995; Loeber, Burke, Lahey, Winters \& Zera, 2000; Veirmeiren, 2003).

$\mathrm{Na}$ análise dos dados qualitativos, os resultados que originaram a subcategoria Dificuldades interpessoais foram concordantes às respostas quantitativas do $Y S R$, ou seja, foi possível verificar tanto no instrumento quanto na entrevista, um estilo de relacionamento interpessoal mais agressivo, descontrole de impulsos, e também, situações que pudessem envolver comportamentos de heteroagressão (Schmitt, Pinto, Gomes, Quevedo \& Stein, 2006; Lühring, Gauer, Vasconcello, Silva \& Navarrete, 2014).

Os problemas internalizantes, como a ansiedade e depressão, isolamento, somatização, pensamento e atenção, não obtiveram resultados considerados clínicos, embora seja possível verificar através da analise de conteúdo (subcategoria aspectos emocionais) uma predominância equivalente de sintomas emocionais negativos. Ansiedade, isolamento, estresse, raiva e irritabilidade foram descritos como sentimentos vivenciados. Esses aspectos podem, ou não, estar relacionados ao uso das drogas ou por exposição de eventos traumáticos durante a vida (Vieira, Aerts, Freddo, Bittencourt, \& Monteiro, 2008). 
Tendo em vista as características interpessoais, ou seja, aquelas habilidades comportamentais que favorecem um relacionamento interpessoal (Del Prette \& Del Prette, 2001; Caballo, 2003; Murta, 2005), a agressividade, raiva, inveja e o isolamento foram listados como dificuldades nas relações (dificuldades interpessoais). 0 ambiente escolar foi descrito como um espaço de construção de amizades, relacionamentos afetivos e estudo, assim como, um ambiente para práticas de consumo de drogas e atos de violência (Galduróz, Noto, Fonseca \& Carlini, 2004).

Os aspectos físicos dos participantes tiveram forte relação com temas frequentemente discutidos pelos jovens dessa faixa etária. Tais comparações auxiliam os jovens na formação da autoestima e do autoconceito (Sisto \& Martinelli, 2004). Chama a atenção um dos adolescentes que relatou estar descontente com cicatrizes que possuía no rosto. Segundo ele, as cicatrizes era resultado de uma agressão física praticada pela sua mãe. Nesse caso, é possível observar aspectos de insatisfação física e de violência intrafamiliar.

A análise dos dados do ambiente familiar apontou a presença do consumo de drogas por parte dos pais. O uso de drogas está frequentemente associado aos relatos de violência física, abuso emocional, posturas autoritárias e situações de abandono, conforme outros levantamentos de outros estudos (Bernardy \& Oliveira, 2010; Cerutti, Ramos, \& Argimon, 2015; Vieira et al., 2008), concretizando a presença de eventos traumáticos na vida dos adolescentes entrevistados. Em contraste disto, o estudo mostrou que todos os participantes relataram sentir saudades da família, atribuindo a eles um significado de importância e valor.

\section{Conclusão}

Os adolescentes avaliados apresentaram comportamentos considerados importantes para um bom repertório nas relações interpessoais e como fatores que podem sugerir menos ajuste emocional e social. Portanto, este estudo pode contemplar os objetivos inicialmente propostos. Foi possível descrever os aspectos sintomatológicos e as características dos relacionamentos interpessoais de cinco adolescentes em tratamento pela dependência de drogas. Habilidades e prejuízos nas relações interpessoais, emoções negativas, positivas e também preocupações com o corpo, ambiente escolar e familiar foram avaliados através da análise de conteúdo de um grupo temático e pelo inventário de comportamentos YSR.

Contudo, nossos dados não permitem a generalização dos achados. Sugere-se aos novos estudos aprimorar o método proposto nessa investigação, incluindo um número maior de participantes e a 
utilização de outros instrumentos que possam ser relacionados às habilidades e aos comportamentos dessa população. Também propomos intervenções de aprimoramento das habilidades interpessoais, de apoio psicológico e estudos que possam refletir acerca do sentido do espaço terapêutico e da reinserção social do adolescente. Por fim, o uso de métodos complementares na avaliação clínica no ingresso do tratamento, por exemplo, pode servir de subsídio para o plano e a prática terapêutica.

\section{Referências}

Achenbach, T. M., \& Rescorla, L. A. (2003). Manual for the ASEBA Adult Forms \& Profiles for ages 18-59. Burlington, VT: University of Vermont, Research Center for Children, Youth \& Families.

Bardin, L. (2009). Análise de Conteúdo. São Paulo: Edições 70.

Bava, S., \& Tapert, S. F. (2010). Adolescent Brain Development and the Risk for Alcohol and Other Drug Problems. Neuropsychology Review, 20, 398-413.

Bernardy, C. C. F., \& Oliveira M. L. F. (2010). O papel das relações familiares na iniciação ao uso de drogas de abuso por jovens institucionalizados. Revista da Escola de Enfermagem USP, 44, 11-17.

Blakemore, S. J., \& Mills, K. L. (2014). Is Adolescence a Sensitive Period for Sociocultural Processing? Annual Review of Psychology, 61, 187-197.

Caballo, V. E. (2003). Manual de avaliação e treinamento das habilidades sociais. São Paulo, Brasil: Santos.

Cerutti, F., Ramos, S. P., \& Argimon, I. I. L. (2015). A implicação das atitudes parentais no uso de drogas na adolescência. Acta Colombiana de Psicología, 18(2), 173-181.

Copeland, J., Rooke, S., \& Swift, W. (2013). Changes in cannabis use among young people: impact on mental health. Current Opinion in Psychiatry, 26, 325-329.

Del Prette, Z. A. P. \& Del Prette, A. (2001). Inventário de habilidades sociais (IHS-DelPrette): Manual de apuração e interpretação. São Paulo, Brasil: Casa do Psicólogo.

Degenhardt, L., Coffey, C., Romaniuk, H., Swift, W., Carlin, J. B., Hall, W. D., \& Patton, G. C. (2013). The persistence of the association between adolescent cannabis use and common mental disorders into young adulthood. Addiction, 108, 124133.

Ferreira, A. C. Z., Capistrano, F. C., Maftum, M. A., Kalinke, L. P., \& Kirchhof, A. L. C. (2012). Caracterização de internações de 
dependentes químicos em uma unidade de reabilitação. Cogitare enfermagem, 17, 444-451.

Fundação Oswaldo Cruz. (2013). Estimativa do número de usuários de crack e/ou similares nas Capitais do País. FIOCRUZ, Rio de Janeiro.

Galduróz, J. C. F., Noto, A. R., Fonseca, A. M., \& Carlini E. A. (2004). $\checkmark$ Levantamento Nacional sobre o Uso de Drogas psicotrópicas entre os estudantes do Ensino fundamental e Médio da Rede publica de Ensino nas 27 Capitais Brasileiras. CEBRID, São Paulo.

Gilbert, R., Widom, C. S., Browne, K., Fergusson, D., Webb, E., \& Janson S. (2009). Burden and consequences of child maltreatment in high-income countries. The Lancet, 373, 6881.

Habigzang, L. F., Diniz, E., \& Koller, S. H. (2014). Trabalhando com adolescentes: teoria e intervenção psicológica. Porto Alegre, Brasil: Artmed.

Hart, S. D., Cox, D. N., \& Hare, R. D. (1995). The Hare Psychopathy Checklist: Screening Version. Toronto, ON: Multi-Health Systems.

Hasler, B. P., Soehner, A. M., \& Clark, D. B. (2014). Circadian rhythms and risk for substance use disorders in adolescence. Current Opinion in Psychiatry, 27, 460-466.

Laranjeira, R., Madruga, C. S., Viana, M. C., Pinsky, I., \& Mitsuhiro, S. (2014). II Levantamento Nacional de Álcool e Drogas violência contra crianças ou adolescentes e uso de drogas. LENAD, São Paulo.

Loeber, R., Burke, J. D., Lahey, B. B., Winters, A., \& Zera, M. (2000). Oppositional defiant and conduct disorder: A review of the past 10 years, part I. Journal of the American Academy of Child \& Adolescent Psychiatry, 39, 1468-1484.

Lucena, C. M. O., \& Ferreira, S. M. S. (2011). Uso de drogas na adolescência: interfaces entre sistema de justiça e sistema de proteção. Revista da Associação Mineira do Ministério Público, 42(25), 26-31.

Lühring, G., Gauer, G. J. C., Vasconcello, D., Silva, L., \& Navarrete, S. S. (2014). Correlação entre traços de psicopatia e abuso de drogas em uma amostra de adolescentes brasileiros em conflito com a lei. Saúde e Desenvolvimento Humano, 2, 29-39.

McClernon, F. J., \& Kollins, S. H. (2008). ADHD and smoking: from genes to brain to behavior. Annals of the New York Academy of Sciences, 1141, 131-147.

Mangerud, W. L., Bjerkeset, O., Holmen, T. L., Lydersen, S., \& Indredavik, M. S. (2014). Smoking, alcohol consumption, and drug use among adolescents with psychiatric disorders 
compared with a population based sample. Journal of Adolescence, 37, 1189-1199.

Martins, M. C., \& Pillon, S. C. (2008). A relação entre a iniciação do uso de drogas e o primeiro ato infracional entre os adolescentes em conflito com a lei. Cad. Saúde Pública, 24, 1112-1120.

Mombelli, M. A., Marcon, S. S., \& Costa, J. B. (2010). Caracterização das internações psiquiátricas para desintoxicação de adolescentes dependentes químicos. Revista brasileira de enfermagem, 63(5), 735-740.

Murta, S. G. (2005). Aplicações do treinamento em habilidades sociais: análise da produção nacional. Psicologia: Reflexão e Crítica, 18, 283-291.

Pechansky, F., Szbot, C. M., \& Scivoletto, S. (2004). Uso de álcool entre adolescentes: conceitos, características epidemiológicas e fatores etiopatogênicos. Revista Brasileira de Psiquiatria, 26, 14-17.

Peeters, M., Vollebergh, W. A. M., Reinout, W. W., \& Field, M. (2014). Psychological Changes and Cognitive Impairments in Adolescent Heavy Drinkers. Alcohol and Alcoholism, 49, 182-186.

Sanchez, Z. M. \& Nappo, S. A. (2002). Sequência de drogas consumidas por usuários de crack e fatores interferentes. Revista de Saúde Pública, São Paulo, Brasil, 36, 420-430.

Sayago, C. B. W., Lucena-Santos, P., Horta, R. L., \& Oliveira, M. S. (2014). Perfil clínico e cognitivo de usuários de crack internados. Psicologia: Reflexão e Crítica, 27, 21-28.

Schmitt, R., Pinto, T. P., Gomes, K. M., Quevedo, J., \& Stein, A. (2006). Personalidade psicopática em uma amostra de adolescentes infratores brasileiros. Archives of Clinical Psychiatry, 33, 297-303.

Schoen-Ferreira, T. H., Aznar-Farias, M., \& Silvares, E. F. M. (2003). A construção da identidade em adolescentes: um estudo exploratório. Estudos de Psicologia, 8, 107-115.

Sisto, F. F., \& Martinelli, S. C. (2004). Estudo preliminar para a construção da escala de autoconceito infanto-juvenil (EAC-IJ). Interação em Psicologia, 8(2), 181-190.

Veirmeiren, R. (2003). Psycopathology and delinquency in adolescents: A descriptive and developmental perspective. Clinical Psychology Review, 23, 277-318.

Vieira, P. C., Aerts, D. R. G. C., Freddo, S. L., Bittencourt, A., \& Monteiro L. (2008). Uso de álcool, tabaco e outras drogas por adolescentes escolares em município do Sul do Brasil. Cadernos de Saúde Pública, 24, 2487-2498.

Wagner, M. F., \& Oliveira, M. S. (2009). Estudo das habilidades sociais em adolescentes usuários de maconha. Psicologia em Estudo, 14, 101-110. 
World Health Organization. (2011). Preventing early pregnancy and poor reproductive outcomes among adolescents in developing countries. WHO, Geneva.

\section{Endereço para correspondência \\ Dhiordan Cardoso da Silva}

Pontifícia Universidade Católica do Rio Grande do Sul

Laboratório de Intervenções Cognitivas do Programa de Pós-Graduação em Psicologia da PUCRS

Avenida Ipiranga, 6681, prédio 11, sala 927, Partenon, CEP 90619-900, Porto

Alegre - RS, Brasil

Endereço eletrônico: dhiordanc@gmail.com

\section{Raquel de Melo Boff}

Pontifícia Universidade Católica do Rio Grande do Sul

Laboratório de Intervenções Cognitivas do Programa de Pós-Graduação em Psicologia da PUCRS

Avenida Ipiranga, 6681, prédio 11, sala 927, Partenon, CEP 90619-900, Porto

Alegre - RS, Brasil

Endereço eletrônico: rmboff@hotmail.com

\section{Maria Isabel Wendling}

Pontifícia Universidade Católica do Rio Grande do Sul

Laboratório de Intervenções Cognitivas do Programa de Pós-Graduação em Psicologia da PUCRS

Avenida Ipiranga, 6681, prédio 11, sala 927, Partenon, CEP 90619-900, Porto Alegre - RS, Brasil

Endereço eletrônico: mariaisabel.wendling@gmail.com

\section{Margareth da Silva Oliveira}

Pontifícia Universidade Católica do Rio Grande do Sul

Laboratório de Intervenções Cognitivas do Programa de Pós-Graduação em Psicologia da PUCRS

Avenida Ipiranga, 6681, prédio 11, sala 927, Partenon, CEP 90619-900, Porto Alegre - RS, Brasil

Endereço eletrônico: marga@pucrs.br

Recebido em: 19/08/2015

Reformulado em: 21/04/2016

Aceito para publicação em: 20/06/2016

\section{Notas}

* Psicólogo Clínico e Pesquisador no Laboratório de Intervenções Cognitivas da Faculdade de Psicologia da PUCRS.

** Psicóloga Clínica, Professora do Centro Universitário UNIVATES e Doutoranda do Programa de Pós-Graduação em Psicologia Clínica da Faculdade de Psicologia da PUCRS.

*** Psicóloga Clínica, Professora da Faculdade de Psicologia da PUCRS e Supervisora do Centro de Estudos da Família e do Indivíduo - CEFI.

**** Psicóloga, Professora Titular do Programa de Pós-Graduação da Faculdade de Psicologia da PUCRS e Coordenadora do Laboratório de Intervenções Cognitivas LABICO. 